\title{
Higgs mass in Noncommutative Geometry
}

\author{
A. Devastato ${ }^{1,2}$, F. Lizzi $^{1,2,3}$, and P. Martinetti ${ }^{1,2}$, \\ ${ }^{1}$ Dipartimento di Fisica, Università di Napoli Federico II \\ 2 INFN, Sezione di Napoli \\ ${ }^{3}$ Departament de Estructura i Constituents de la Matèria, Universitat de Barcelona
}

\begin{abstract}
In the noncommutative geometry approach to the standard model, an extra scalar field $\sigma$ - initially suggested by particle physicist to stabilize the electroweak vacuum - makes the computation of the Higgs mass compatible with the $126 \mathrm{GeV}$ experimental value. We give a brief account on how to generate this field from the Majorana mass of the neutrino, following the principles of noncommutative geometry.
\end{abstract}

Talk given by P.M. at Corfou Workshop on noncommutative field theory and gravity, september 2013

The idea that the Higgs field is somehow related to a noncommutativity of spacetime emerged in the late 80 '- early 90' [10, 14] and reached its full achievement with Connes and al [3, 9] description of the Standard Model of elementary particles [SM] in terms of noncommutative geometry [NCG]. The latter is a generalization of Riemannian geometry, that allows to incorporate in a single geometrical object the gravitational degrees of freedom (the commutative part of the geometry) and the quantum ones (the noncommutative part). The central object in this description is a generalized Dirac operator $D$, whose components are the usual Dirac matrices, the Yukawa couplings of the fermions and the mixing parameters for quarks and neutrinos. The SM Lagrangian minimally coupled to the Einstein-Hilbert action (in Euclidean signature) is retrieved from one single action formula. Furthermore, the mass of the Higgs boson comes as a function of the other parameters of the theory, thus can be calculated. The model has been through various improvements [6], but the prediction was always around $m_{H}=170 \mathrm{GeV}$, a value ruled out by Tevratron in 2008.

The discovery of a $126 \mathrm{GeV}$ Higgs boson in summer 2012 at LHC raised the question of the stability of the electroweak vacuum: the quartic selfcoupling in the Higgs potential becomes negative at high energy, indicating the vacuum is not stable but metastable (fig. 11). There does not seem to be a consensus whether this is a real problem or not: on the one hand the lifetime of this metastable state is longer than the age of the universe, on the other hand metastability may have some cosmological consequence, it seems unlikely that at early age the Higgs field has been trapped everywhere in the false vacuum, and in some firewall scenario the metastability might even have catastrophic consequences (there is a vast literature on the subject, see e.g. [12] and references within for a recent account). Furthermore, $126 \mathrm{Gev}$ is very close to the stability zone (fig. 2), suggesting that new physics may be around the corner. This instability can be cured by a new scalar field $\sigma$ coupled to the Higgs (e.g. [15]). As a bonus, by taking into account this field in the description of the SM in NCG, it is possible to bring the value of $m_{H}$ to $126 \mathrm{GeV}$, without modifying the fermion contain of the SM [5] (using an extra scalar field in NCG to lower $m_{H}$ was already in [19], but required new fermions). The point is thus to understand how to obtain $\sigma$ intrinsically within the NCG framework.

In [5] this is done by turning into a field the (constant) Majorana mass term $k_{R}$ of the neutrino, which is one of the component of the generalized Dirac operator $D$. However the substitution $k_{R} \rightarrow \sigma k_{R}$ is somehow ad-hoc: why should this - and only this - component of $D$ become a field? In this proceeding we give a non-technical summary of our recent proposal [13] on how to obtain $\sigma$ within the NCG framework. We also discuss our result, in particular in the light of another proposal made almost simultaneously in [7, 8].

agostino.devastato@na.infn.it, fedele.lizzi@na.infn.it, martinetti@na.infn.it 


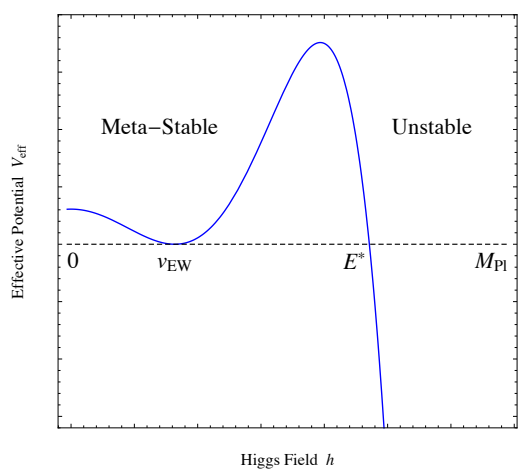

FIG. 3: Schematic of the effective potential $V_{\text {eff }}$ as a function of the Higgs field $h$. This is not drawn to scale; for a Higgs mass in the range indicated by $\mathrm{LHC}$ data, the heirarchy is $v_{E W} \ll E^{*} \ll M_{\mathrm{P}}$, where each of these 3 energy scales is separated by several orders of magnitude.

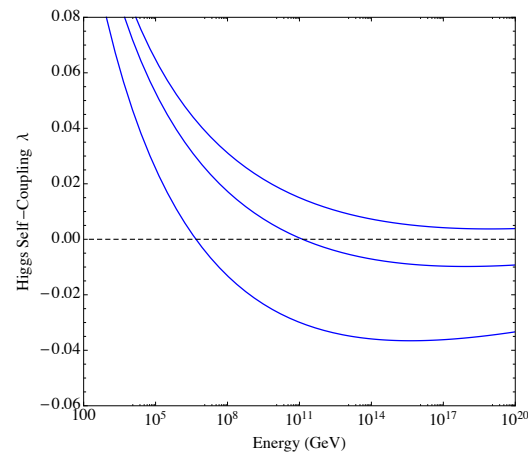

FIG. 2: Higgs self-coupling $\lambda$ as a function of energy, for different values of the Higgs mass from 2-loop RG evolution. Lower curve is for $m_{H}=116 \mathrm{GeV}$, middle curve is for $m_{H}=126 \mathrm{GeV}$, and upper curve is for $m_{H}=130 \mathrm{GeV}$. All other Standard Model couplings have been fixed in this plot, including the top mass at $m_{t}=173.1 \mathrm{GeV}$.

Fig. 1 Higgs quartic selfcoupling and the effective potential (from [17]).

A spectral triple is a $*$-algebra $\mathcal{A}$ that acts faithfully on a Hilbert space $\mathcal{H}$, together with an operator $D$ on $\mathcal{H}$ such that $[D, a]$ is bounded and $a[D-\kappa \mathbb{I}]^{-1}$ is compact for all $a \in \mathcal{A}$ and $\kappa \notin \operatorname{Sp} D$. With some extra-conditions that are the algebraic version of the geometrical properties of a Riemannian manifold, and that include the definition of two more operators (a chirality $\Gamma$ and a real structure $J$ ), Connes showed [11] that to any spectral triple $(\mathcal{A}, \mathcal{H}, D)$ with $\mathcal{A}$ unital and commutative is associated a compact Riemannian spin manifold $\mathcal{M}$ such that $\mathcal{A}=C^{\infty}(\mathcal{M})$. These conditions easily adapt to the noncommutative case. The ones that are important for the present work are the grading, the order 0 and the order 1 conditions:

$$
[\Gamma, a]=0, \quad\left[a, J b J^{-1}\right]=0, \quad\left[[D, a], J b J^{-1}\right]=0 \quad \forall a, b \in \mathcal{A} .
$$
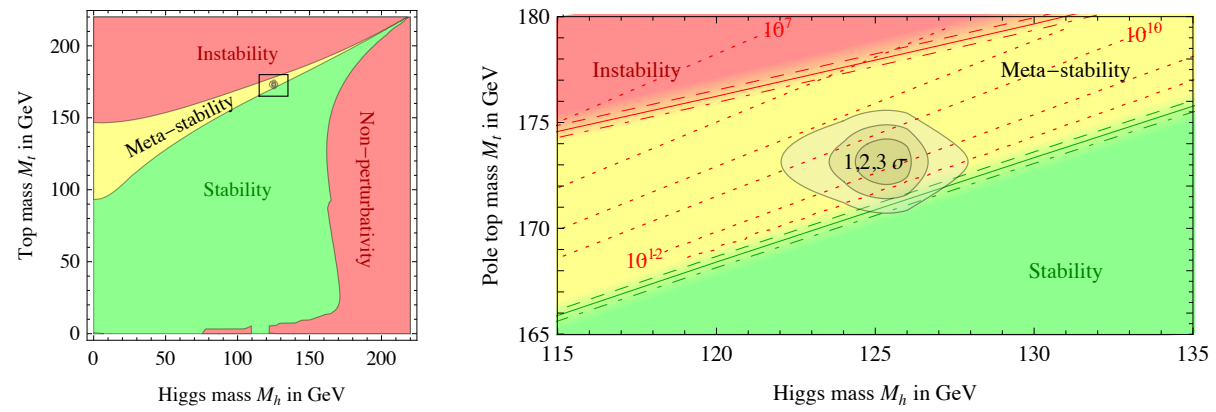

Fig. 2 Stability of the electroweak vacuum in the $m_{\text {top }}, m_{H}$ plane (from [12]).

The spectral triple of the SM is the product of the spectral triple $\left(C^{\infty}(\mathcal{M}), L^{2}(\mathcal{M}, S), \not \partial\right)$ of a compact Riemannian manifold $\mathcal{M}$ (here $L^{2}(\mathcal{M}, S)$ is the Hilbert space of spinors and $\not \partial$ is the usual Dirac operator) by a finite dimensional spectral triple

$$
\begin{aligned}
\mathcal{A}_{s m}=\mathbb{C} \oplus \mathbb{H} \oplus M_{3}(\mathbb{C}), \quad \mathcal{H}_{F}=\mathbb{C}^{N \times 32}=\mathcal{H}_{R} \oplus \mathcal{H}_{L} \oplus \oplus \mathcal{H}_{R}^{c} \oplus \mathcal{H}_{L}^{C} \\
D_{F}=\left(\begin{array}{cccc}
0_{8 N} & M & M_{R} & 0_{8 N} \\
M^{\dagger} & 0_{8 N} & 0_{8 N} & 0_{8 N} \\
M_{R}^{\dagger} & 0_{8 N} & 0_{8 N} & \bar{M} \\
0_{8 N} & 0_{8 N} & M^{T} & 0_{8 N}
\end{array}\right) \quad N=\sharp \text { generations. }
\end{aligned}
$$


The Hilbert space $\mathcal{H}_{R}=\mathbb{C}^{N \times 8}$ is the space of right fermions ( 6 colored quarks +1 lepton +1 neutrino), $\mathcal{H}_{L}$ is the space of left fermions and $\mathcal{H}_{R}^{C}, \mathcal{H}_{L}^{c}$ are the ones of antifermions. The matrix $M$ contains the quarks, leptons, neutrinos (Dirac) mass with CKM mixing; $M_{R}$ contains the Majorana neutrinos mass $k_{R}$. The chirality is $\gamma_{F}=\operatorname{diag}\left(\mathbb{I}_{8 N},-\mathbb{I}_{8 N},-\mathbb{I}_{8 N}, \mathbb{I}_{8 N}\right)$ and the real structure is an antidiagonal matrix with entries $\mathbb{I}_{16 N}, \mathbb{I}_{16 N}$. The total spectral triple is

$$
\mathcal{A}=C^{\infty}(\mathcal{M}) \otimes \mathcal{A}_{s m}, \quad \mathcal{H}=L^{2}(\mathcal{M}, S) \otimes \mathcal{H}_{F}, \quad D=\not \partial \otimes \mathbb{I}_{N \times 32}+\gamma^{5} \otimes D_{F}
$$

with $\Gamma=\gamma^{5} \otimes \gamma_{F}$ and $J=\mathcal{J} \otimes J_{F}$, where $\mathcal{J}$ is the charge conjugation operator.

The gauge fields of the SM (including the Higgs) are obtained by fluctuation of the Dirac operator substituting $D$ with

$$
D_{A}=D+A+J A J^{-1}
$$

where $A$ is a generalized gauge potential, i.e. a selfadjoint element of the form $\sum_{i} a_{i}\left[D, b_{i}\right]$ with $a_{i}, b_{i} \in \mathcal{A}$. This amounts to turning the constant components of $M$ in $D_{F}$ into fields on the manifold $\mathcal{M}$. The spectral action $\operatorname{Tr} f\left(\frac{D_{A}}{\Lambda}\right)$ with $f$ a smooth approximation of the characteristic function of the interval $[0,1]$ and $\Lambda$ an energy scale, then yields the bosonic part of the Lagrangian of the SM minimally coupled with EinsteinHilbert action. It requires a unique unification scale $\Lambda$. With $\Lambda=10^{17} \mathrm{GeV}$, the running of the Higgs quartic selfcoupling $\lambda$ under the big desert hypothesis yields $m_{H}=\sqrt{2 \lambda} v_{E W} \simeq 170 \mathrm{GeV}$. After having turned into a field $k_{R} \sigma$ the neutrino Majorana mass $k_{R}$, the same procedure allows to pull back $m_{H}$ to $126 \mathrm{GeV}$.

One may think to obtain $\sigma$ as the other bosonic fields, i.e. thanks to a fluctuation of the metric. Unfortunately the order 1 condition prevents this. Denoting $D_{R}$ the part of $D_{F}$ that contains only the neutrino mass, one has

$$
\left[\left[D_{R}, a\right], J b J^{-1}\right]=0 \quad \forall a, b \in \mathcal{A}_{s m} \Longrightarrow\left[D_{R}, a\right]=0 .
$$

In other terms, because of the first order condition there is no way to obtain $\sigma$ by a fluctuation of the Majorana part of the finite dimensional Dirac operator $D_{F}$.

In [13] we proposed to obtain $\sigma$ starting from a bigger algebra than the one of the SM. Under natural assumptions (irreducibility of the representation, existence of a cyclic vector), technical requirements of the NCG model (there is a representation of the opposite algebra that commutes with the action of the algebra and is implemented by an operator that commutes with the chirality) and a hypothesis on the role of quartenion, one has that the most general finite dimensional algebra satisfying the axiom of noncommutative manifolds is of the form [4]: $M_{a}(\mathbb{H}) \oplus M_{2 a}(\mathbb{C}), a \in \mathbb{N}$, and acts on an Hilbert space of dimension $d=2 \times(2 \times a)^{2}$. The case $a=1$ is too small to get the gauge group of the SM as the group of unitaries of $M(\mathbb{H}) \oplus M_{2}(\mathbb{C})$. The next choice $a=2$ yields $d=32$, that is the number of fermions per generation. As explained in [4], the grading condition imposes the reduction

$$
M_{2}(\mathbb{H}) \oplus M_{4}(\mathbb{C}) \longrightarrow \mathcal{A}_{L R}=\mathbb{H}_{L} \oplus \mathbb{H}_{R} \oplus M_{4}(\mathbb{C}) .
$$

The order 1 condition and neutrino mass further imposes

$$
\mathcal{A}_{L R} \longrightarrow \mathbb{H}_{L} \oplus \mathbb{H}_{R} \oplus M_{3}(\mathbb{C}) \oplus \mathbb{C} \longrightarrow \mathbb{H}_{L} \oplus \mathbb{C}^{\prime} \oplus M_{3}(\mathbb{C}) \oplus \mathbb{C}
$$

with $\mathbb{C}=\mathbb{C}^{\prime}$. Hence the the reduction of $\mathcal{A}_{F}$ to the algebra $\mathcal{A}_{s m}$ of the standard model.

The case $a=3$ yields $d=72$. There is no obvious relation with the 32 particles/generation of the SM. Interestingly, $a=4$ yields $d=128$, which is 4 times the number of particles/generation. Viewing 4 as the number of components of a Dirac spinor on a 4-dimensional manifold, one can thus decompose the total Hilbert space (for 1 generation), using the fermion doubling of the model [18], as

$$
\mathcal{H}=L^{2}(\mathcal{M}, S) \otimes \mathcal{H}_{F}=L^{2}(\mathcal{M}) \otimes \mathrm{H}_{F} \quad \text { where } \quad \mathrm{H}_{F}=\mathbb{C}^{4} \otimes \mathcal{H}_{F}=\mathbb{C}^{4} \otimes \mathbb{C}^{32}=\mathbb{C}^{128}
$$


In writing 9 we ignore global obstruction and assume that the r.h.s. equality of the first equation above holds on a local trivialization of the spin bundle. The idea we want to promote is that by mixing the spin degrees of freedom ( $s=l, r$ for the left, right components of a Dirac spinor, $\dot{s}=\dot{0}, \dot{1}$ for the (anti)-particles ones) with the internal degrees of freedom

$$
\begin{aligned}
& C=p, a \quad \text { (particle, antiparticles), } \quad I=0,1,2,3 \quad \text { (lepto-color), } \\
& \alpha=u_{R}, d_{R}, u_{L}, d_{L}(I=1,2,3), e_{R}, \nu_{R}, e_{L}, \nu_{L}(I=0) \quad \text { (flavor), }
\end{aligned}
$$

then the Hilbert space $\mathcal{H}$ of the standard model is big enough to represent the grand algebra $(a=4)$

$$
\mathcal{A}_{G}=M_{4}(\mathbb{H}) \oplus M_{8}(\mathbb{C})
$$

(tensorized by $C^{\infty}(\mathcal{M})$ ) without touching the SM particle contents, and satisfying the order 0 condition.

Explicitly the representation is as follows. We denote a spinor in $\mathcal{H}$ as $\Psi_{s \dot{s} \alpha}^{C I}$ and view both $Q \in M_{4}(\mathbb{H})$ and $M \in M_{8}(\mathbb{C})$ as $2 \times 2$ block matrices, with block $4 \times 4$ complex matrices:

$$
Q=\left(\begin{array}{cc}
Q_{\dot{0} \alpha}^{\dot{0} \beta} & Q_{\dot{0} \alpha}^{\mathrm{i} \beta} \\
Q_{\dot{1} \alpha}^{0 \beta} & Q_{\dot{1} \alpha}^{1 \beta}
\end{array}\right), \quad M=\left(\begin{array}{ll}
M_{r J}^{r I} & M_{r J}^{l I} \\
M_{l J}^{r I} & M_{l J}^{l I}
\end{array}\right) .
$$

Viewing the components of the matrices in 13 as functions on $\mathcal{M}$, an element $A=(Q, M)$ in $C^{\infty}(\mathcal{M}) \otimes$ $\mathcal{A}_{G}$ acts on $\mathcal{H}$ as

$$
A_{s \dot{s} D J \alpha}^{t \dot{t C I \beta}}=\left(\delta_{0}^{C} \delta_{s}^{t} \delta_{J}^{I} Q_{\dot{s} \alpha}^{\dot{t} \beta}+\delta_{1}^{C} M_{s J}^{t I} \delta_{\dot{s}}^{t} \delta_{\alpha}^{\beta}\right) .
$$

The indices $\beta, J$ run on the same set as $\alpha, I$. The Dirac operator, chirality and real structure are unchanged. The grading condition imposes the reduction

$$
\mathcal{A}_{G}=M_{4}(\mathbb{H}) \oplus M_{8}(\mathbb{C}) \longrightarrow \mathcal{A}_{G}^{\prime}=\left(M_{2}(\mathbb{H})_{L} \oplus M_{2}(\mathbb{H})_{R}\right) \oplus\left(M_{4}(\mathbb{C})_{l} \oplus M_{4}(\mathbb{C})_{r}\right) .
$$

A solution of the first-order condition of the Majorana Dirac operator only, $\gamma^{5} \otimes D_{R}$, is

$$
\mathcal{A}_{G}^{\prime} \longrightarrow \mathcal{A}_{G}^{\prime \prime}=\left(\mathbb{H}_{L} \oplus \mathbb{H}_{L}^{\prime} \oplus \mathbb{C}_{R} \oplus \mathbb{C}_{R}^{\prime}\right) \oplus\left(\mathbb{C}_{l} \oplus M_{3}(\mathbb{C})_{l} \oplus \mathbb{C}_{r} \oplus M_{3}(\mathbb{C})_{r}\right)
$$

with $\mathbb{C}_{R}=\mathbb{C}_{r}=\mathbb{C}_{l}$. The main result of [13] is that for $A \in \mathcal{A}_{G}^{\prime \prime}$

$$
\left[\gamma^{5} \otimes D_{R}, A\right] \text { is not necessarily zero. }
$$

In other terms, starting from the grand algebra one can generate the field $\sigma$ by a fluctuation of the Majorana mass term which respects the first order condition imposed by the Majorana mass term.

The further reduction to the standard model, that is $\mathbb{C}_{R}^{\prime}=\mathbb{C}_{R}, \mathbb{H}_{L}^{\prime}=\mathbb{H}_{L}, M_{3}(\mathbb{C})_{l}=M_{3}(\mathbb{C})_{r}$, is obtained by the first order condition on the non-Majorana part of the Dirac operator.

Let us now discuss our result. The representation $(13)$ of $C^{\infty}(\mathcal{M}) \otimes \mathcal{A}_{G}$ together with the Dirac operator in (4) do not yield a spectral triple, because whatever $A \in C^{\infty}(\mathcal{M}) \otimes \mathcal{A}_{G}$, the operator

$$
\left[\not \partial \otimes \mathbb{I}_{32}, A\right]=P+T^{\mu} \partial_{\mu}
$$

( $P$ and $T^{\mu}$ are matrices whose explicit form is given in [13]) is not bounded. It becomes bounded if $T^{\mu}=0$ for all $\mu=1, . ., 4$, but this precisely means that the action of the grand algebra should be diagonal on the spinorial indices, meaning the reduction of $\mathcal{A}_{G}$ to $\mathcal{A}_{s m}$. Thus the mixing of the spin and internal indices has two consequences:

- the bounded bosonic operators $B$ generated by a fluctuation of the Dirac operator include the field $\sigma$;

- there is a "deeper alteration of spacetime", encoded within the unbounded operator $T^{\mu} \partial_{\mu}$. 
This last point is the most open to drastic changes which may be imagined, for example like dropping out the associativity of the algebra [16].

Alternatively, one may imagine a cosmological scenario beginning with a "pre-geometric phase", described by the grand algebra and the finite dimensional Dirac operator $\gamma^{5} \otimes D_{R}$. The right neutrino would then play the role of a "primary elementary particle", that generates the field $\sigma$. Then usual geometry (encoded within the free Dirac operator $\not \partial \otimes \mathbb{I}$ ) emerges at a later stage, and provokes the reduction to the SM. This makes and interesting echo to a recent inflationary interpretation of the field $\sigma$ [2]. Moreover very recent data [1] seem to indicate an inflationary scale at a scale of $10^{16} \mathrm{GeV}$, a scale in broad agreement with the unification of the coupling constant required by this approach. From a more mathematical point of view, we stress that the triple $\left(C^{\infty}(\mathcal{M}) \otimes \mathcal{A}_{G}, \mathcal{H}, \gamma^{5} \otimes D_{R}\right)$ satisfies the bounded commutator condition, but $\gamma^{5} \otimes D_{R}$ has no compact resolvent.

To summarize, the grand algebra transfers the problem of generating $\sigma$ from the noncommutative to the commutative part of the geometry: with the algebra of the standard model, $C^{\infty}(\mathcal{M}) \otimes \mathcal{A}_{s m}$, the first order condition is always satisfied by the free Dirac operator, the problem is all in $D_{R}$. Using the grand algebra, we have that $\gamma^{5} \otimes D_{R}$ both generates the field $\sigma$ and satisfies the first-order condition. But the free Dirac does not satisfies this condition (neither the bounded commutator one). Of course this is not satisfactory but this suggests interesting path to explore.

Another question is whether the reduction to the SM imposed by the first order condition can be understood dynamically (i.e. by a minimization of the spectral action), as in the model of Chamseddine, Connes and van Suijlekom where $\sigma$ is generated from $\mathcal{A}_{s m}$ by a fluctuation without first order condition.

Acknowledgements The authors thank W. van Suijlekom for discussion.

\section{References}

[1] P.A.R.Ade et al. [ BICEP2 Collaboration], BICEP2 I: Detection Of B-mode Polarization at Degree Angular Scales, arXiv:1403.3985 [astro-ph.CO]; BICEP2 II: Experiment and Three-Year Data Set, arXiv:1403.4302 [astro-ph.CO].

[2] I. Bars, P. J. Steinhardt, and N. Turok, Cyclic cosmology, conformal symmetry and the metastability of the higgs, Preprint arXiv 1307.8106 (2013).

[3] A. H. Chamseddine and A. Connes, The spectral action principle, Commun. Math. Phys. 186 (1996), 737-750.

[4] A. H. Chamseddine and A. Connes, Why the standard model ?, J. Geom. Phys 58 (2008), 38-47.

[5] A. H. Chamseddine and A. Connes, Resilience of the spectral standard model, JHEP 104 (2012).

[6] A. H. Chamseddine, A. Connes, and M. Marcolli, Gravity and the standard model with neutrino mixing, Adv. Theor. Math. Phys. 11 (2007), 991-1089.

[7] A. H. Chamseddine, A. Connes, and Walter van Suijlekom, Beyond the spectral standard model: emergence of Pati-Salam unification, JHEP 1311 (2013), 132.

[8] A. H. Chamseddine, A. Connes, and Walter van Suijlekom, Inner fluctuations in noncommutative geometry without first order condition, J. Geom. Phy. 73 (2013).

[9] A. Connes, Gravity coupled with matter and the foundations of noncommutative geometry, Commun. Math. Phys. 182 (1996), 155-176.

[10] A. Connes, J. Lott, Particle models and noncommutative geo., Nuclear Phys. B Proc. Suppl. 18B (1990), $29-47$.

[11] Alain Connes, On the spectral characterization of manifolds, J. Noncom. Geom. 7 (2013), no. 1, 1-82.

[12] G. Degrassi, S. Di Vita, J. Elias-Miro, J. R. Espinosa, G. F. Giudice, G. Isidori, and A. Strumia, Higgs mass and vacuum stability in the standard model at nnlo, JHEP 08 (2012) 098.

[13] A. Devastato, F. Lizzi, P. Martinetti, Grand Symmetry, Spectral Action and the Higgs mass, JHEP 01 (2014) 042.

[14] M. Dubois-Violette, J. Madore, and R. Kerner, Classical bosons in a noncommutative geometry, Class. Quantum Grav. 1709 (1989).

[15] J. Elias-Miro, J. R. Espinosa, G. F. Giudice, H. M. Lee, and A. Strumia. Stabilization of the electroweak vacuum by a scalar threshold effect, JHEP 06 (2012) 031.

[16] S. Farnsworth and L. Boyle, Non-associative geometry and the spectral action principle, Preprint arXiv 1303.1782 (2013).

[17] M. P. Hertzberg, A correlation between the higgs mass and dark matter, (2012), arXiv: 1210.3624.

[18] F. Lizzi, G. Mangano, G. Miele and G. Sparano, Fermion Hilbert space and fermion doubling in the noncommutative geometry approach to gauge theories, Phys. Rev. D 55 (1997) 6357 [hep-th/9610035].

[19] C. A. Stephan, New scalar fields in noncommutative geometry, Phys. Rev. D 79 (2009).

[20] T. Schücker, Higgs mass predictions, arXiv 0708.3344 [hep-th] 